\title{
Development and prevention of advanced diabetic nephropathy in RAGE-overexpressing mice
}

\author{
Yasuhiko Yamamoto, ${ }^{1,2}$ Ichiro Kato, ${ }^{2}$ Toshio Doi, ${ }^{3}$ Hideto Yonekura, ${ }^{1}$ Seiji Ohashi, ${ }^{3}$ \\ Masayoshi Takeuchi, ${ }^{4}$ Takuo Watanabe, ${ }^{1,5}$ Sho-ichi Yamagishi, ${ }^{1}$ Shigeru Sakurai, ${ }^{1}$ \\ Shin Takasawa, ${ }^{2}$ Hiroshi Okamoto, ${ }^{2}$ and Hiroshi Yamamoto ${ }^{1}$
}

\begin{abstract}
${ }^{1}$ Department of Biochemistry and Molecular Vascular Biology, Kanazawa University Graduate School of Medical Science, Kanazawa, Japan

${ }^{2}$ Department of Biochemistry, Tohoku University Graduate School of Medicine, Sendai, Japan

${ }^{3}$ Department of Laboratory Medicine, Tokushima University School of Medicine, Tokushima, Japan

${ }^{4}$ Department of Biochemistry, Faculty of Pharmaceutical Science, Hokuriku University, Kanazawa, Japan

${ }^{5}$ Department of Stem Cell Biology, Kanazawa University Graduate School of Medical Science, Kanazawa, Japan

Address correspondence to: Hiroshi Yamamoto, Department of Biochemistry and Molecular Vascular Biology,

Kanazawa University Graduate School of Medical Science, 13-1 Takara-machi, Kanazawa 920-8640, Japan.

Phone: 81-76-265-2180; Fax: 81-76-234-4226; E-mail: yamamoto@med.kanazawa-u.ac.jp.
\end{abstract}

Received for publication November 13, 2000, and accepted in revised form May 29, 2001.

Vascular complications arising from multiple environmental and genetic factors are responsible for many of the disabilities and short life expectancy associated with diabetes mellitus. Here we provide the first direct in vivo evidence that interactions between advanced glycation end products (AGEs; nonenzymatically glycosylated protein derivatives formed during prolonged hyperglycemic exposure) and their receptor, RAGE, lead to diabetic vascular derangement. We created transgenic mice that overexpress human RAGE in vascular cells and crossbred them with another transgenic line that develops insulin-dependent diabetes shortly after birth. The resultant double transgenic mice exhibited increased hemoglobin $A_{1 c}$ and serum AGE levels, as did the diabetic controls. The double transgenic mice demonstrated enlargement of the kidney, glomerular hypertrophy, increased albuminuria, mesangial expansion, advanced glomerulosclerosis, and increased serum creatinine compared with diabetic littermates lacking the RAGE transgene. To our knowledge, the development of this double transgenic mouse provides the first animal model that exhibits the renal changes seen in humans. Furthermore, the phenotypes of advanced diabetic nephropathy were prevented by administering an AGE inhibitor, ( \pm )-2-isopropylidenehydrazono-4-oxo-thiazolidin-5-ylacetanilide (OPB-9195), thus establishing the AGE-RAGE system as a promising target for overcoming this aspect of diabetic pathogenesis.

J. Clin. Invest. 108:261-268 (2001). DOI:10.1172/JCI200111771.

\section{Introduction}

Nephropathy is a life-threatening complication of diabetes mellitus and is the leading cause of endstage renal disease (ESRD) in developed countries (1). Approximately $30 \%$ of insulin-dependent diabetes mellitus (IDDM) patients suffer from diabetic nephropathy $(1,2)$, eventually undergoing renal dialysis or transplantation. The features characteristic of this disease include persistent albuminuria, a progressive decline in renal function, and, histopathologically, mesangial expansion followed by glomerulosclerosis. To understand the pathogenesis of diabetic nephropathy and to develop preventive and therapeutic measures against it, suitable animal models are needed. However, there has been no single animal model that develops the renal changes seen in humans (3). Spontaneously diabetic animals such as nonobese diabetic (NOD) mice develop only limited lesions, this being, at most, mild mesangial sclerosis (4). The same is the case with chemically induced diabetic rodents (5).
Exposure of proteins to reducing sugars like glucose results in nonenzymatic glycation, which forms reversible Schiff bases and Amadori compounds (6). A series of further complex molecular rearrangements then yields irreversible advanced glycation end products (AGEs) (6). In diabetes, prolonged hyperglycemia superdrives this reaction and AGEs accumulate in the circulating blood and in various tissues (6). AGE infusion studies in experimental animals have suggested a potential role of AGEs in the pathogenesis of diabetic nephropathy $(7,8)$ as well as retinopathy $(9)$. Receptor-dependent mechanisms are likely to work in the AGE-induced tissue dysfunction. Receptor for AGE (RAGE) is the best-characterized cell surface molecule to which AGEs bind, and it has been shown to be expressed in a variety of cell types (10-15). Recent in vitro studies demonstrated that AGEs elicit vascular cell changes typical of diabetes. These include angiogenic and thrombogenic responses of endothelial cells (ECs) $(12,13)$, a decrease in pericytes, (14) and the enhanced synthesis of ECM proteins by 

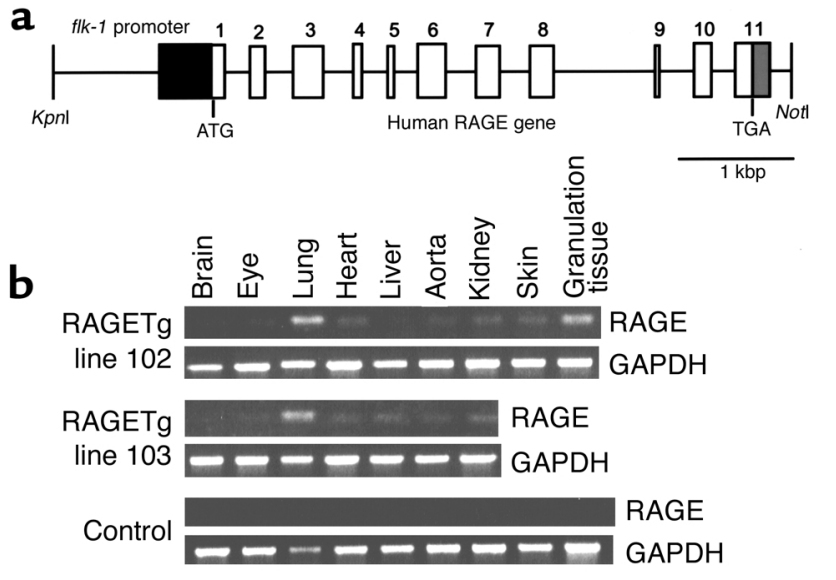

c
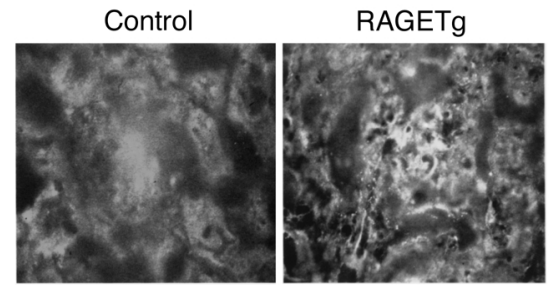

d
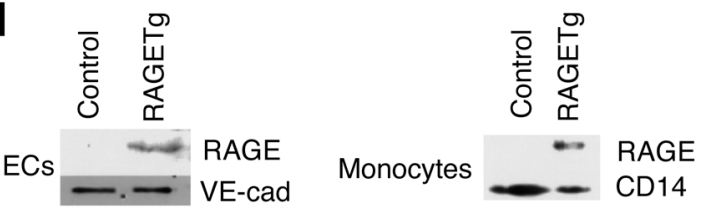

\section{Figure 1}

Generation and characterization of RAGE transgenic mice. (a) Transgene construct. The human RAGE genomic sequence, beginning with the initiator codon in exon 1 and ending 129 bp downstream from the last exon, was placed under the transcriptional control of the murine flk-1 promoter. The flk-1 fragment encompassed the $5^{\prime}$ untranslated region (dark gray box), sharing the ATG codon with the RAGE fragment. (b) Transgene-derived transcripts. Total RNAs isolated from various tissues of line 102 and 103 heterozygotes were analyzed by RT-PCR. The granulation tissue - the focus of angiogenesis - was prepared by punching out an area of the dorsal skin about 4-5 mm in diameter. The PCR-amplified products had a chain length of $354 \mathrm{bp}$ as predicted and were sequence-verified. (c) Immunofluorescence staining of kidneys from line 102 RAGETg or nontransgenic control at 4 months of age using antihuman RAGE-specific polyclonal Ab. Original magnification, $\times 430$. (d) Translation products of the transgene. Extracts of isolated ECs from renal cortex or peripheral blood monocytes of line 102 RAGETg or the nontransgenic control were immunodetected with the human RAGE Ab. Specific bands were marked at $55 \mathrm{kDa}$ in line 102 RAGETg EC and monocyte extracts. VE-cad, VE-cadherin, i.e., an EC marker; CD14, a monocyte marker.

renal mesangial cells (15). All of these changes are RAGE-dependent, as evidenced by their cessation by RAGE antisense or ribozyme (12-15).

In the present study, we constructed transgenic mice that overexpress human RAGE in vascular cells (RAGETg). When these mice were made diabetic by crossbreeding with another transgenic mouse deficient in the islet production of insulin (16), the resultant double transgenic animals developed renal insufficiency and advanced glomerulosclerosis that resembled human diabetic nephropathy. This in vivo approach has thus established the functional importance of the AGE-RAGE system in the development of diabetic nephropathy, and the RAGE-overexpressing IDDM mice are regarded as the first single animal model in which the process of diabetes-induced kidney changes leading to ESRD can be followed. The AGE-RAGE system would seem to be a promising target for the prophylaxis and therapy of diabetic complications, and a study with ( \pm )-2-isopropylidenehydrazono-4-oxo-thiazolidin-5-ylacetanilide (OPB-9195) (17) included in this paper suggests that the inhibition of AGE formation could be an effective remedy.

\section{Methods}

Construction of transgenic mice and induction of diabetes. A mouse flk-1 promoter previously reported to be active in vascular ECs in vitro was used (18). The $0.9-\mathrm{kbp}$ fragments of the mouse flk-1 promoter and the 3.4$\mathrm{kbp}$ fragments of the entire exonic and intronic region of the human RAGE gene (provided by T. Ikemura and K. Sugaya, National Institute of Genetics, Mishima, Japan) were ligated in the correct orientation by a modified polymerase chain reaction method (19). Primers used in the PCR reactions were $5^{\prime}$-GAGGTACCAGTTCACAACCGAAATGTCTTCTAGGG- ${ }^{\prime}$ and $5^{\prime}$ CTGCTGTTCCGGCTGCCATCCTGCACCTCGCGCTGGGCA-3' (nucleotides -623 to $-597,+280$ to +299 ) for mouse flk-1 (18), and 5'-ATGGCAGCCGGAACAGCAGTT- $3^{\prime}$ and $5^{\prime}$-GAGCGGCCGCATCTTCTCCCCAACTTGAGTAGA-3' (nucleotides 6665-6685, 10027-10049) for human RAGE gene (20), containing KpnI and NotI sites (underlined sequences), respectively. The resultant 4.3-kbp hybrid gene was microinjected into fertilized eggs as described (16). Six out of 24 newborn offspring carried the transgene, as detected by PCR analyses of DNA from tail tissue using primers for flk-1 promoter $\left(5^{\prime}\right.$-AGGGACGGAGAAGGAGT- $3^{\prime}:+240$ to +256) (18) and human RAGE gene (5'-TCACCCCACAGACTGAG-3': 6704-6720) (20).

RAGETg was crossbred with another transgenic mouse carrying human cDNA for inducible nitric oxide synthase (iNOS) under the control of the insulin promoter (iNOS$\mathrm{Tg})(16)$. There was no expression of iNOS protein in the kidney (16). The resultant four groups of male littermates were used for analysis after PCR verification of the transgenes. Each group was fed either normal or 0.28\% OPB9195 mixed mouse chow (provided by Fujii Memorial Research Institute, Otsuka Pharmaceutical, Ohtsu, Japan) from 1 to 6 months after birth. The levels of blood glucose and hemoglobin $\mathrm{A}_{1 \mathrm{c}}\left(\mathrm{HbA}_{1 \mathrm{c}}\right)$ were measured from tail vein blood using Dexter $Z$ sensor and DCA2000 analyzer (Bayer Medical, Tokyo, Japan) (21), respectively.

The procedures were approved by the Institutional Animal Care and Use Committee guidelines at Tohoku University and at Kanazawa University Graduate School of Medical Science. 
$R T-P C R$. Total RNA was isolated from various tissues, and from isolated renal glomeruli, of transgenic or control mice using the guanidinium thiocyanate method, and reverse-transcribed (22). The isolation of glomeruli from mouse kidneys was performed as described (23). Primer sequences for human RAGE mRNA detection were $5^{\prime}$-AAGCCCCTGGTGCCTAATGAG-3' and 5'-CACCAATTGGACCTCCTCCA-3' (nucleotides 508-528 and 728-747 in GenBank AB036432); those for mouse RAGE mRNA detection were 5'-CCTGGGTGCTGGTTCTTGCTCT- $3^{\prime}$ and 5'-GATCTGGGTGCTCTTACGGTCC-3' (nucleotides 31-52 and 1209-1230 in GenBank L33412); those for mouse GAPDH mRNA detection were the same as described (24). The amounts of total RNA templates (100 ng) and the numbers for amplification cycles ( 35 cycles for human RAGE and 30 cycles for mouse RAGE and GAPDH) were chosen in quantitative ranges. An aliquot of each RT-PCR product was electrophoresed on $2 \%$ agarose gel containing ethidium bromide.

Isolation of ECs and monocytes, and Western blot analysis. ECs from mouse renal cortex were cultivated for 2 days in a gelatin-coated plate after isolation by specific selection with rat anti-mouse CD31/PECAM-1 mAb-coated (MEC7.46; HyCult Biotechnology b.v.) magnetic beads (Dynabeads M-450 sheep anti-rat IgG; Dynal, Oslo, Norway) as described (25). Monocytes were isolated from peripheral blood by Ficoll-Paque (Pharmacia Biotech AB, Uppsala, Sweden) centrifugation followed by magnetic cell sorter (MACS; Miltenyi Biotec, Bergisch Gladbach, Germany) using CD11b/Mac-1 $\alpha$ Ab-coated MicroBeads (Miltenyi Biotec) according to the manufacturer's instructions (26). Cells were homogenized in PBS, 1\% NP-40, 0.5\% deoxycholate, $10 \mathrm{mM}$ EDTA, 0.1\% SDS, and $1.0 \mathrm{mM}$ PMSF, and centrifuged at 15,000 $\mathrm{g}$ for 30 minutes at $4{ }^{\circ} \mathrm{C}$. Supernatants were used for protein assay. Protein concentrations were determined using Coomassie Protein Assay Reagents (Pierce Chemical Co., Rockford, Illinois, USA) by the method of Bradford (27). Proteins were electrophoresed on 10\% SDS-PAGE and transferred electrophoretically to polyvinilidene difluoride membranes. Membranes were blocked with 5\% nonfat dry milk in PBS and $0.1 \%$ Tween-20, and then incubated with 1:100 dilution of goat anti-vascular endothelial-cadherin (anti-VE-cadherin) polyclonal $\mathrm{Ab}$ (Santa Cruz Biotechnology Inc., Santa Cruz, California, USA), 1:100 dilution of goat anti-mouse CD14 polyclonal Ab (Santa Cruz Biotechnology Inc.), or $0.25 \mu \mathrm{g} / \mathrm{ml}$ of immunoaffinity-purified anti-RAGE polyclonal Ab raised against 16-amino-acid synthetic peptides (QRRQRRGEERKAPENQ) that corresponded to amino acid residues 364-379 of the human RAGE protein (11). After incubation with 1:2,000 dilution of anti-goat or anti-rabbit IgG conjugated to horseradish peroxidase, the immune complexes were visualized with an enhanced chemiluminescence detection system (ECL; Amersham Pharmacia Biotech, Piscataway, New Jersey, USA).
Determination of AGE concentration. Serum $N^{\varepsilon}$-carboxymethyl-lysine (CML) and non-CML AGEs were differentially determined using a competitive ELISA as described (28). One $\mathrm{U} / \mathrm{ml}$ of CML or non-CML AGEs corresponded to a protein concentration of $1 \mu \mathrm{g} / \mathrm{ml}$ CML-BSA or non-CML AGE-BSA, respectively.

Determination of urine albumin/creatinine ratio and serum creatinine. Urinary or serum creatinine was measured by the Jaffe reaction (29). The level of urinary albumin was measured by ELISA with sheep anti-mouse albumin as described $(4,30)$. The ratio of urinary albumin to creatinine was then calculated.

Renal histology and morphometric analyses. Kidneys were processed for light microscopy examination, and the severity of the renal sclerosis was scored by multiple analysts on an arbitrary scale from 0 to $4(4,31,32)$. The mean glomerular volume was determined as described previously $(4,30)$. To quantify mesangial expansion, periodic acid-Schiff-positive (PAS-positive) area in the mesangium was determined as mesangium area using an image analyzer with a microscope (Image Processor for Analytical Pathology; Sumitomo Chemical Co., Tokyo, Japan) (33). Immunofluorescence analysis was carried out as described $(30,34)$ using an affinity-purified polyclonal $\mathrm{Ab}$ specific to human RAGE raised against 10-amino-acid synthetic peptides (PLVPNEKGVS) corresponding to amino acid residues 163-172 of the human RAGE protein (11), anti-CML $\mathrm{Ab}$, and anti-non-CML AGE Ab (28).

\section{Results}

Expression of RAGE transgene. We created RAGETg by introducing into fertilized ova of C57BL/6J $\times \mathrm{CBA} / \mathrm{J} \mathrm{F}_{1}$ mice a transgene carrying human RAGE genomic DNA under the control of the murine $f l k-1$ promoter, which a

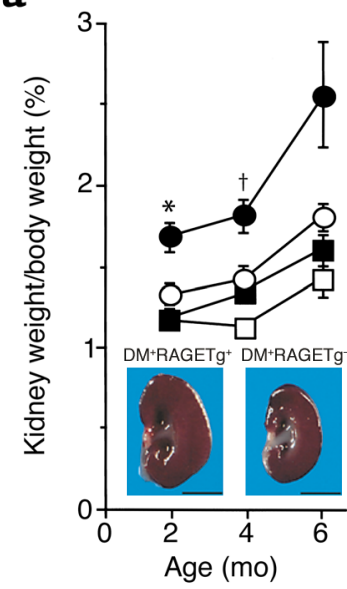

b

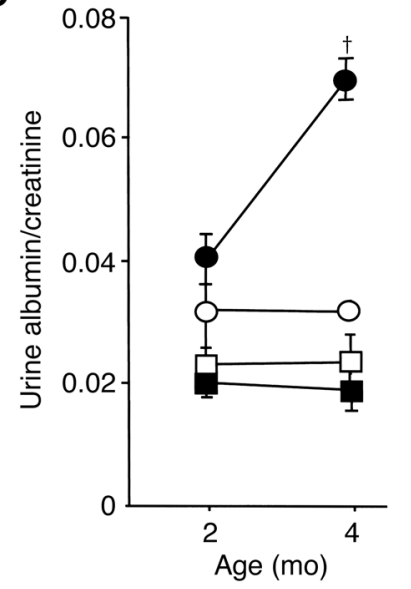

Figure 2

Renal changes in the early stage. (a) Kidney weight/body weight ratio. Inset: Sagittal section of the kidney at 4 months of age. Bar, $5 \mathrm{~mm}$. (b) Albuminuria. Filled circles, $\mathrm{DM}^{+} \mathrm{RAGETg} \mathrm{g}^{+}$; open circles, $\mathrm{DM}^{+} \mathrm{RAGETg}^{-}$; filled squares, DM-RAGETg ${ }^{+}$; open squares, DM-RAGETg-. Data are mean \pm SEM. ${ }^{*} P<0.02,{ }^{\dagger} P<0.05$ compared with $\mathrm{DM}^{+} \mathrm{RAGETg}$. Statistical analysis was performed by $t$ test. 


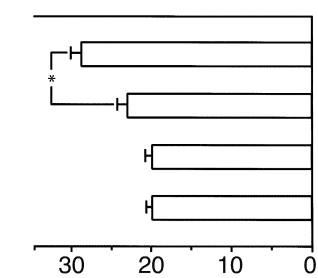

DM+RAGETg $^{+}$
DM+RAGETg-
DM-RAGETg
DM-RAGETg-

Interglomerular cell number

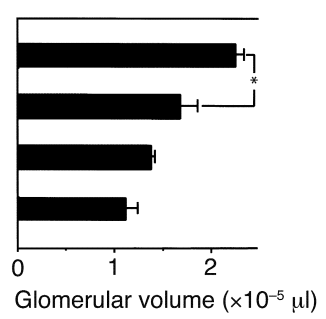

\section{Figure 3}

Glomerular changes in the early stage. Glomerular cell proliferation (left) and glomerular volume (right) at 2 months of age. Data are mean \pm SEM. ${ }^{*} P<0.05$. Statistical analysis was performed by $t$ test.

acts in ECs (18) (Figure 1a). PCR analysis revealed that five out of six potentially transgenic mice exhibited transgene transmission into their germlines. Two independent lines, 102 and 103, carrying high copy numbers, were used for subsequent experiments. RT-PCR analysis with human RAGE-specific primers revealed active transcription of the transgene in the lung, skin granulation tissue (the focus of angiogenesis), kidney, heart, aorta, and eyes of each line, but not in controls (Figure 1b). Immunofluorescent examination of kidney sections demonstrated that glomeruli of RAGETg were positively stained for human RAGE in an EC pattern, but not in the control (Figure 1c). Immunoblotting of cell extracts from renal cortical ECs demonstrated the overexpression of RAGE proteins in RAGETg (Figure 1d). Expression of the RAGE protein was also detected in monocytes from peripheral blood of RAGETg (Figure 1d).

Induction of diabetes. There are several means to induce diabetes in experimental animals $(35,36)$. Chemical or surgical maneuvers for diabetes induction might, however, cause some diversity among individual animals in terms of the extent of severity and the onset of diabetes. Accordingly, we employed a genetic approach by which a diabetic state and advanced glycation as well would be most stably induced. That is, RAGETg was crossbred with iNOSTg that consistently develops IDDM as early as 1 week after birth due to the iNOS-mediated selective destruction of insulin-producing pancreatic $\beta$ cells (16). RAGETg was back-crossed to the nontransgenic parental strain CD-1 of iNOSTg for 4 or 5 generations to unify the genetic background. Then, the heterozygotes of male iNOSTg and female RAGETg were mated, thereby yielding four groups of littermates at five or six generations with CD-1 background, which carried both, either, or neither of the transgenes. They were tentatively designated $\mathrm{DM}^{+} \mathrm{RAGETg}^{+}$, $\mathrm{DM}^{+} \mathrm{RAGETg}^{-}, \mathrm{DM}^{-} \mathrm{RAGETg}^{+}$, and DM-RAGETg-, since the mice carrying the iNOS transgene exclusively developed hyperglycemia regardless of the presence or absence of the RAGE transgene. Blood analysis revealed sustained hyperglycemia and high $\mathrm{HbA}_{1 \mathrm{c}}$ levels in the former two groups but not in the latter two (Table 1). There were no significant differences in these indices between $\mathrm{DM}^{+} \mathrm{RAGETg}^{+}$and $\mathrm{DM}^{+} \mathrm{RAGETg}^{-}$(Table 1). Body weight was also invariant between the two groups at all time points tested during the 6-month observation period (data not shown). To determine the levels of circulating AGEs, serum was differentially measured by competitive ELISA for CML (derivative of AGEs mainly formed by peroxidation) and for non-CML AGEs (28). Serum non-CML AGE levels rapidly increased with time in both $\mathrm{DM}^{+} \mathrm{RAGETg}^{+}$and $\mathrm{DM}^{+} \mathrm{RAGETg}{ }^{-}$mice, but without significant differences between them at each time point (Table 1). There was a tendency of higher serum CML levels in the diabetic groups than in the nondiabetic groups, but without significant differences (Table 1). These findings were comparable to observations in human diabetic and nondiabetic subjects (28). With the 103 line, similar results were obtained by blood glucose, $\mathrm{HbA}_{1 \mathrm{c}}$, and serum AGE assays (data not shown).

Phenotypic changes of the kidney. The kidney weight/body weight ratios from $\mathrm{DM}^{+} \mathrm{RAGETg}{ }^{+}$showed a marked increase when compared with age-matched $\mathrm{DM}^{+} \mathrm{RAGETg}^{-}$mice (Figure 2a). The urinary albumin/creatinine ratio became significantly higher in $\mathrm{DM}^{+} \mathrm{RAGETg}^{+}$than in the other groups at 4 months (Figure 2b). The microscopic lesions noted in the diabetic groups consisted of glomerular cell proliferation, glomerular hypertrophy, mesangial expansion, and glomerulosclerosis. $\mathrm{DM}^{+} \mathrm{RAGETg}^{+}$showed accelerated increases in glomerular cell proliferation and glomeru-
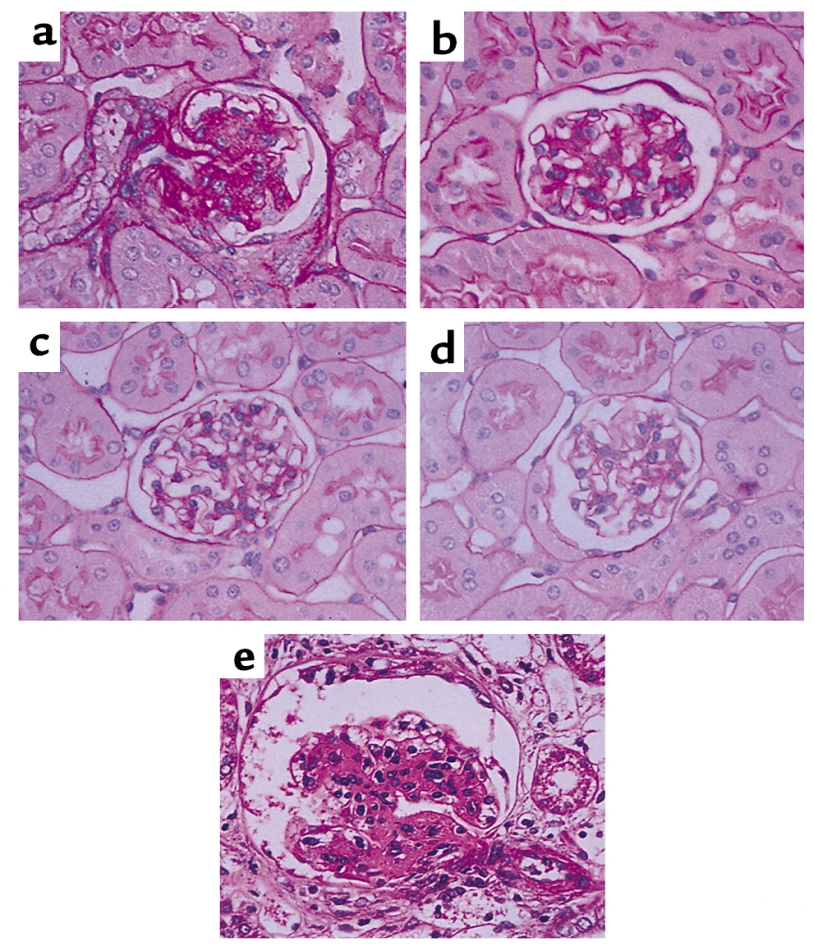

Figure 4

Renal changes in the late stage. PAS staining of thin kidney sections from line $102 \mathrm{DM}^{+} \mathrm{RAGETg}^{+}(\mathbf{a}), \mathrm{DM}^{+} \mathrm{RAGETg}^{-}(\mathbf{b}), \mathrm{DM}^{-\mathrm{RAGETg}}{ }^{+}$ (c), and DM-RAGETg (d) at 4 months of age and $\mathrm{DM}^{+} \mathrm{RAGETg}^{+}(\mathbf{e})$ at 8 months of age. Original magnification, $\times 670$. 
Table 1

Diabetes-related blood indices in line 102

\begin{tabular}{|c|c|c|c|c|c|c|}
\hline Group & $\begin{array}{l}\text { Age } \\
(\mathrm{mo})\end{array}$ & $\begin{array}{l}\text { Mice } \\
(n)\end{array}$ & $\begin{array}{l}\text { Blood glucose } \\
\qquad(\mathrm{mg} / \mathrm{dl})\end{array}$ & $\begin{array}{c}\mathrm{HbA1c} \\
(\%)\end{array}$ & $\begin{array}{l}\mathrm{CML} \\
(\mathrm{U} / \mathrm{ml})\end{array}$ & $\begin{array}{l}\text { Non-CML AGEs } \\
(\mathrm{U} / \mathrm{ml})\end{array}$ \\
\hline \multirow[t]{3}{*}{$\mathrm{DM}^{+} \mathrm{RAGETg}^{+}$} & 2 & 4 & $509 \pm 61$ & $8.0 \pm 0.6$ & $4.1 \pm 0.4$ & $10.3 \pm 0.4$ \\
\hline & 4 & 3 & $609 \pm 94$ & $9.5 \pm 0.1$ & $3.7 \pm 0.6$ & $11.9 \pm 1.0$ \\
\hline & 6 & 7 & $547 \pm 13$ & $8.3 \pm 0.6$ & $7.7 \pm 0.1$ & $24.2 \pm 1.3$ \\
\hline OPB & 6 & 8 & $693 \pm 26$ & $9.4 \pm 0.5$ & $5.4 \pm 0.4^{\mathrm{A}}$ & $14.2 \pm 0.8^{C}$ \\
\hline \multirow[t]{3}{*}{$\mathrm{DM}^{+} \mathrm{RAGETg}^{-}$} & 2 & 6 & $482 \pm 22$ & $8.2 \pm 0.4$ & $3.4 \pm 0.5$ & $7.3 \pm 2.3$ \\
\hline & 4 & 5 & $447 \pm 45$ & $9.1 \pm 0.5$ & $5.1 \pm 0.8$ & $14.4 \pm 2.5$ \\
\hline & 6 & 9 & $503 \pm 19$ & $7.5 \pm 0.8$ & $7.3 \pm 0.6$ & $20.7 \pm 2.5$ \\
\hline OPB & 6 & 5 & $586 \pm 74$ & $8.9 \pm 0.9$ & $4.3 \pm 1.0^{B}$ & $10.2 \pm 2.6^{\mathrm{D}}$ \\
\hline \multirow[t]{3}{*}{ DM-RAGETg $^{+}$} & 2 & 6 & $177 \pm 5$ & $3.2 \pm 0.2$ & $2.9 \pm 0.6$ & $2.5 \pm 1.0$ \\
\hline & 4 & 6 & $157 \pm 3$ & $3.8 \pm 0.3$ & $3.3 \pm 0.6$ & $6.4 \pm 1.6$ \\
\hline & 6 & 11 & $134 \pm 4$ & $<2.5$ & $7.6 \pm 0.3$ & $20.2 \pm 1.7$ \\
\hline OPB & 6 & 4 & $145 \pm 16$ & $<2.5$ & $6.3 \pm 1.0$ & $13.2 \pm 3.7$ \\
\hline \multirow[t]{3}{*}{ DM-RAGETg- $^{-}$} & 2 & 3 & $188 \pm 8$ & $3.3 \pm 0.1$ & $2.6 \pm 1.0$ & $2.8 \pm 0.6$ \\
\hline & 4 & 5 & $157 \pm 4$ & $3.4 \pm 0.1$ & $3.0 \pm 0.1$ & $4.4 \pm 0.5$ \\
\hline & 6 & 11 & $125 \pm 5$ & $<2.5$ & $6.2 \pm 0.4$ & $12.6 \pm 1.0$ \\
\hline OPB & 6 & 10 & $169 \pm 9$ & $<2.5$ & $5.0 \pm 0.7$ & $8.7 \pm 1.1$ \\
\hline
\end{tabular}

Data are expressed as the mean \pm SEM. OPB, oral OPB-9195 administration for 5 months. ${ }^{A} P<0.002$ and ${ }^{B} P<0.05$ compared with CML values of DM ${ }^{+} R A G E T g{ }^{+}$ and $\mathrm{DM}^{+} \mathrm{RAGETg}^{-}$at 6 months of age without OPB-9195 treatment, respectively. ${ }^{C} P<0.002$ and ${ }^{D} P<0.05$ compared with non-CML AGE values of DM ${ }^{+} \mathrm{RAGETg}^{+}$ and DM+RAGETg at 6 months of age without OPB-9195 treatment, respectively. Statistical analysis was performed by $t$ test. A high non-CML AGE level was noted in DM-RAGETg ${ }^{+}$at 6 months; the reason for this may be partly explained by hemolysis.

lar volume in comparison with DM+RAGETg- mice (Figure 3). Diffuse glomerulosclerosis progressed as the mice aged in both groups. However, there was a conspicuous difference in the severity of mesangial expansion and glomerulosclerosis at 4 months between $\mathrm{DM}^{+} \mathrm{RAGETg}^{+}$ and $\mathrm{DM}^{+} \mathrm{RAGETg}^{-}$, as evidenced by increased accumulation of PAS-positive materials in the mesangial area of the former (Figure 4, a-d). Quantitative examinations of at least 50 glomeruli per mouse revealed significant increases in mesangium area, mesangium fraction (relative mesangium area calculated as mesangium area/glomerular area ratio) (Figure 5), and sclerosis index in $\mathrm{DM}^{+} \mathrm{RAGETg}^{+}$compared with $\mathrm{DM}^{+} \mathrm{RAGETg}^{-}$mice at 4 months of age (Figure 6a). Immunostaining showed that significant amounts of not only non-CML AGEs but also CML accumulated in the mesangial area in the diabetic mice, but not in nondiabetic mice (Figure 7, a-d). At 6 months of age, the serum creatinine level of $\mathrm{DM}^{+} \mathrm{RAGETg}^{+}$increased to $1.24 \pm 0.07 \mathrm{mg} / \mathrm{dl}$, being the highest among the groups (Figure 6a). Further, typical nodular lesions and hyaline arteriosclerosis were noted at 8 months of age in $\mathrm{DM}^{+}$RAGETg ${ }^{+}$(Figure 4e). Since hypertension has been known to be another risk factor for progressive glomerular disease (37), the blood pressure was monitored. Though $\mathrm{DM}^{+} \mathrm{RAGETg}{ }^{+}$showed a slightly higher value at 6 months of age, there was no statistically significant difference in systolic blood pressure among the four groups $\left(\mathrm{DM}^{+} \mathrm{RAGETg}{ }^{+}, 135 \pm 8 \mathrm{mmHg}\right.$; $\mathrm{DM}^{+} \mathrm{RAGETg}^{-}, 108 \pm 10 \mathrm{mmHg} ; \mathrm{DM}^{-} \mathrm{RAGETg}^{+}, 109 \pm 9$ mmHg; DM-RAGETg-, $108 \pm 10 \mathrm{mmHg}$ ). Experiments with the line 103 series revealed a similar tendency in all of the above indices.

Upregulation of endogenous mouse RAGE $m R N A$ in diabetes. Since AGE ligands themselves have been shown to induce the transcription of the RAGE gene (38), we determined the endogenous mouse RAGE mRNA levels along with the transgene-derived human RAGE mRNA levels in renal glomeruli by RT-PCR with mouse- or human-specific primer pairs. The endogenous RAGE mRNA levels were increased in glomeruli of $\mathrm{DM}^{+} \mathrm{RAGETg}^{+}$since 2 months of age and in DM $^{+}$RAGETg ${ }^{-}$too at 4 months of age (Figure $7 \mathrm{e}$ ). On the other hand, the transgene expression under the control of flk-1 promoter was invariant under diabetic conditions (Figure 7e).

Suppression of diabetic nephropatby by OPB-9195. We then conducted an intervention study with OPB-9195, a thiazolidine derivative that can inhibit AGE formation by blocking carbonyl groups on glycation intermediates (17). When the mice had received OPB-9195 orally for 5 months, the serum non-CML and CML AGE levels of both $\mathrm{DM}^{+} \mathrm{RAGETg}^{+}$and $\mathrm{DM}^{+} \mathrm{RAGETg}^{-}$were significantly reduced to levels similar to those of nondiabetic mice, with blood glucose and $\mathrm{HbA}_{1 \mathrm{c}}$ being essentially unaffected (Table 1). The OPB-9195 treatment signifi-

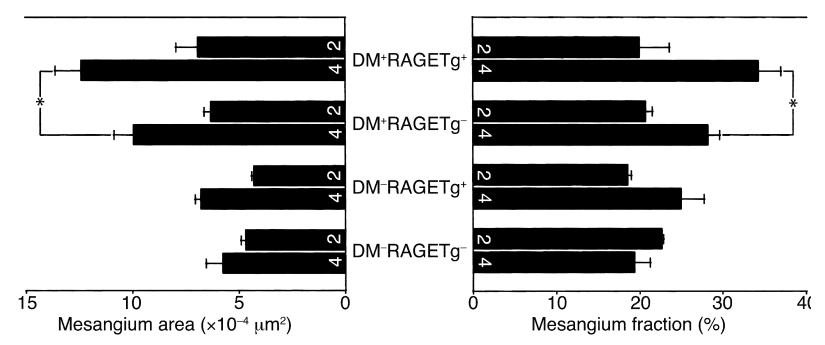

Figure 5

Mesangial expansion. Mesangium area (left) and mesangium fraction (right). Numbers on columns indicate months of age. Data are mean \pm SEM. ${ }^{*} P<0.05$. Statistical analysis was performed by $t$ test. 
a

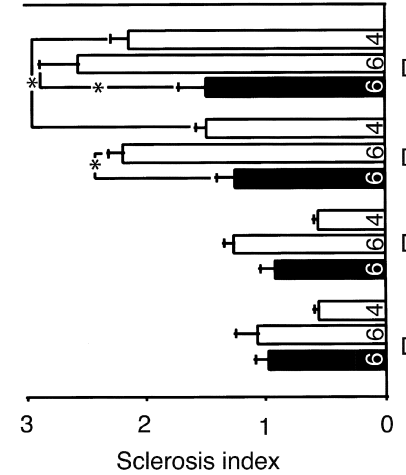

b

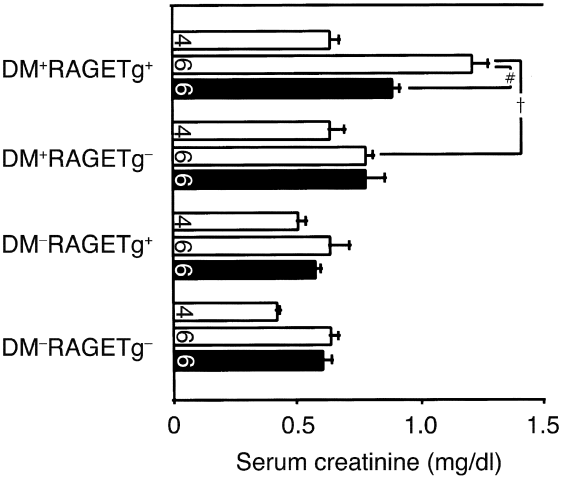

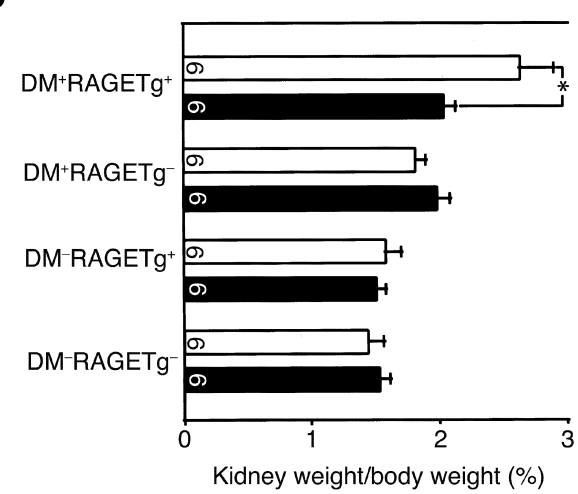

Figure 6

(a) Sclerosis index and serum creatinine level. (b) Kidney weight/body weight ratio. Open bars, animals not treated with OPB-9195; filled columns, animals treated with OPB-9195. Numbers on columns indicate months of age. Data are mean \pm SEM. ${ }^{*} P<0.05,+P<0.005$, ${ }^{\#} P<0.001$. Statistical analysis was performed by $t$ test.

cantly suppressed the increases in the kidney weight/body weight ratio (Figure $6 \mathrm{~b}$ ) and the sclerosis index and serum creatinine (Figure $6 \mathrm{a}$ ) of $\mathrm{DM}^{+} \mathrm{RAGETg}^{+}$ at 6 months of age. A similar tendency was observed in experiments with the line 103 series (data not shown).

\section{Discussion}

In its early phase, diabetic nephropathy is characterized by increased albuminuria, glomerular hypertrophy, and nephromegaly (37). Mesangial expansion, glomerulosclerosis, and increased serum creatinine then follow in the late phase (37). In this study, the diabetic mouse itself, which we used to mate with the RAGETg, showed glomerular hypertrophy until 4 months of age and progressive mesangial expansion and glomerulosclerosis afterward. Furthermore, overexpression of human RAGE gene in ECs, which normally were only faintly stained for RAGE protein in renal arteries, arterioles, and glomerular capillaries (39-41), was found to result in a significant acceleration of all of the early- and late-phase indices of diabetic nephropathy. Because the extent of this acceleration was roughly proportional to the degree of RAGE transgene expression, and because circulating levels of AGEs and their deposition in renal glomeruli were essentially invariant between the IDDM animals carrying or not carrying the RAGE transgene, it was considered to be the level of RAGE expression that had ratelimited the progression of diabetic nephropathy. The higher expression of RAGE induced the earlier and higher expression of endogenous mouse RAGE in renal glomeruli under diabetic conditions (Figure 7e). The upregulation of the endogenous RAGE would then superdrive the development of the renal lesions in $\mathrm{DM}^{+} \mathrm{RAGETg}^{+}$. In addition, oral administration of a thi-
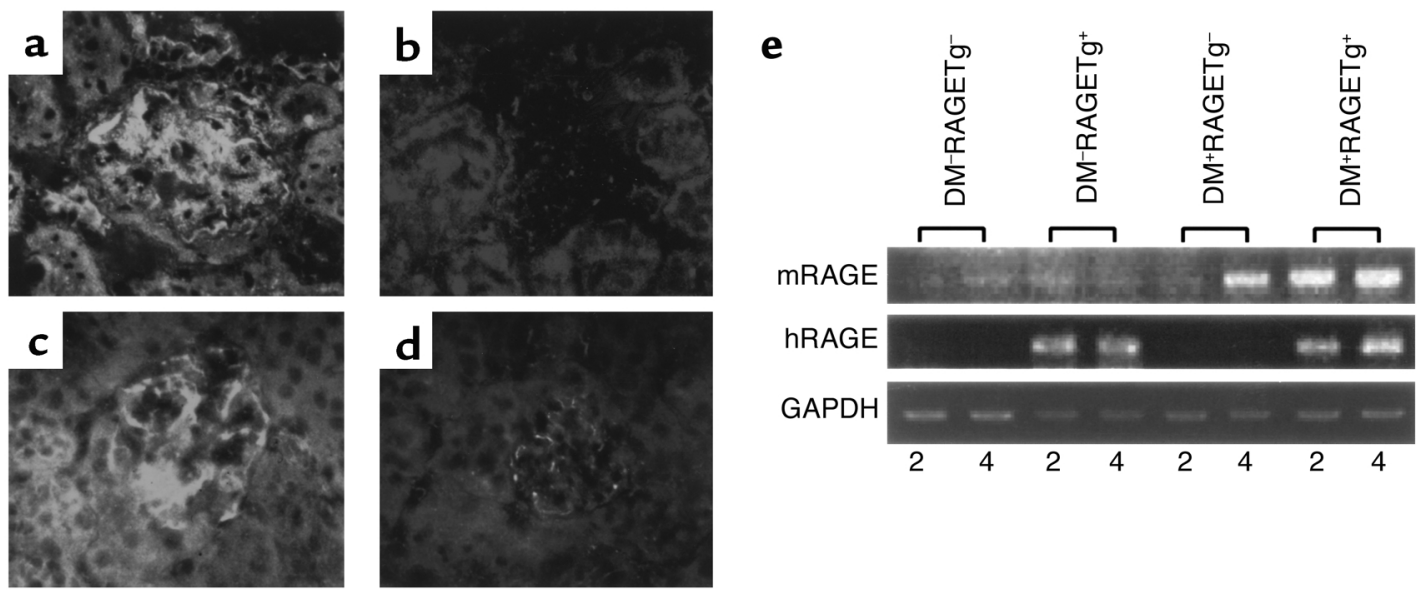

Figure 7

Stain for non-CML AGEs ( $\mathbf{a}$ and $\mathbf{b})$ and CML (c and $\mathbf{d})$ in the kidneys from 4-month-old line $102 \mathrm{DM}^{+} \mathrm{RAGETg}^{+}(\mathbf{a}$ and $\mathbf{c})$ and DM-RAGETg(b and $\mathbf{d}$ ) mice. Glomeruli were stained for non-CML AGEs and CML in DM+RAGETg to extents similar to those in DM ${ }^{+}$RAGETg ${ }^{+}$but were much less stained in DM-RAGETg ${ }^{+}$and DM-RAGETg $g^{-}$mice. Original magnification, $\times 330$. (e) Upregulation of mouse RAGE mRNA in diabetic glomeruli. Total RNAs isolated from glomeruli of each of the four groups in line 102 were analyzed by RT-PCR. mRAGE, mouse RAGE mRNA; hRAGE, human RAGE mRNA. Numbers indicate months of age. 
azolidine compound, OPB-9195, significantly suppressed the development of the renal insufficiency, nephromegaly, and glomerulosclerosis in $\mathrm{DM}^{+} \mathrm{RAGETg}^{+}$ without affecting the levels of blood glucose or $\mathrm{HbA}_{1 \mathrm{c}}$. OPB-9195 is known to inactivate carbonyl intermediates of nonenzymatic glycation with an activity about one order of magnitude more potent than that of aminoguanidine (17). The prevention of diabetic nephropathy by OPB-9195 in the transgenic mice was associated with a decrease in AGEs, though this could reflect other hitherto unknown properties of the drug. These results indicate that endothelial RAGE and its engagement by AGEs contribute to the full expression of diabetic nephropathy, and that the AGE-RAGE system could become a target for intervention in this disease.

Then, how does the AGE-RAGE interaction lead to such kidney changes? It is reported that AGE-RAGE interactions upregulate the production of various cytokines and growth factors such as TNF- $\alpha$ (42), PDGF (22), and VEGF (12) via oxidant stress formation. TNF- $\alpha$ and VEGF are known to increase vascular permeability and to diminish barrier properties (43). This may underlie the increased albuminuria. AGERAGE interactions also have been shown to stimulate endocytosis and transcytosis of AGEs in ECs (44), thus probably causing the abluminal accumulation of AGEs, to which mural cells like mesangial cells would then be exposed. AGE-induced oxidant stress might also accelerate CML production in situ in the glomeruli (45). The exposure to AGEs has been shown to be followed by the transcriptional induction of the mesangial cell synthesis of type IV collagen, a major component of the expanded ECM in diabetic nephropathy $(46,47)$. This induction was also proved to be mediated by RAGE (15). In addition, positive staining for type I and IV collagen was noted in the glomeruli of the present nephropathy model (data not shown). Moreover, EC expression of VCAM-1 is also known to be induced by AGE engagement of RAGE (48), enhancing mononuclear cell adhesion to the capillary wall, which would allow macrophage invasion into the mesangial area. This may provide an additional source of cytokines such as TGF- $\beta$ that can contribute to the development of glomerulosclerosis. The flk-1 promoter employed to construct RAGETg in this work is known to also work in the monocyte-macrophage lineage (49). The RAGE overexpression in monocytes (Figure $1 \mathrm{~d}$ ) is considered to be consistent with this notion and may augment the interaction with AGE on invaded macrophages, thereby contributing to accelerating the progression of nephropathy in the present model. RAGE engagement by endogenous ligands, namely amphoterin (50) and EN-RAGE (S100/calgranulins; ref. 51), is reportedly involved in the network formation of cerebral neurons and in proinflammatory reactions, respectively. The S100/calgranulins are known to be released from activated inflammatory cells, including mononuclear phagocytes as well as polymorphonuclear leukocytes and lymphocytes (52). The immuno- fluorescence analysis of the kidney sections revealed that renal glomeruli were stained for S100/calgranulins in diabetic $\left(\mathrm{DM}^{+} \mathrm{RAGETg}^{+}\right.$and $\mathrm{DM}^{+} \mathrm{RAGETg}-$ but not in nondiabetic (DM-RAGETg ${ }^{+}$and DM-RAGETg ${ }^{-}$) groups, the heaviest stain being marked in $\mathrm{DM}^{+} \mathrm{RAGETg}^{+}$(data not shown). This suggests that the diabetic kidney contains two RAGE ligands, both nonenzymatically glycated adducts and S100/calgranulin proteins, and that S100/calgranulins accumulating in the kidney lesions could further activate ECs and monocytes/macrophages via their engagement of RAGE. AGEs are senescent proteins that accumulate during prolonged diabetic exposure, and can also engage cell surface RAGE, this resulting in the deterioration of vascular functions $(12,15,53)$. Diabetic complications appear to exploit molecular devices primarily evolved for development and survival.

\section{Acknowledgments}

The authors thank S. Matsudaira, R. Kitamura, T. Yachi, and Y. Shichinohe for assistance, S. Kim and Y. Ouchi (University of Tokyo) for blood pressure measurement, and B. Bell for reading the manuscript. This work was supported in part by the "Research for the Future" Program of Japan Society for the Promotion of Science (grant 97L00805), by a grant-in-aid from the Ministry of Education, Culture, Sports, Science, and Technology, Japan (no. 12770054), and by a research fund from the Hokuriku Industrial Advancement Center.

1. Bojestig, M., Arnqvist, H.J., Hermansson, G., Karlberg, B.E., and Ludvigsson, J. 1994. Declining incidence of nephropathy in insulin-dependent diabetes mellitus. N. Engl. J. Med. 330:15-18.

2. Krolewski, M., Eggers, P.W., and Warram, J.H. 1996. Magnitude of endstage renal disease in IDDM: a 35 year follow-up study. Kidney Int. 50:2041-2046

3. Velasquez, M.T., Kimmel, P.L., and Michaelis, O.E., IV. 1990. Animal models of spontaneous diabetic kidney disease. FASEB J. 4:2850-2859.

4. Doi, T., et al. 1990. Glomerular lesions in nonobese diabetic mouse: before and after the onset of hyperglycemia. Lab. Invest. 63:204-212.

5. Williamson, J.R., et al. 1987. Increased vascular permeability in spontaneously diabetic $\mathrm{BB} / \mathrm{W}$ rats and in rats with mild versus severe streptozotocin-induced diabetes. Prevention by aldose reductase inhibitors and castration. Diabetes. 36:813-821.

6. Brownlee, M., Cerami, A., and Vlassara, H. 1988. Advanced glycosylation endproducts in tissue and the biochemical basis of diabetic complications. N. Engl. J. Med. 318:1315-1321.

7. Vlassara, H., et al. 1994. Advanced glycosylation end products induce glomerular sclerosis and albuminuria in normal rats. Proc. Natl. Acad. Sci. USA. 91:11704-11708.

8. Yang, C.W., et al. 1994. Advanced glycation end products up-regulate gene expression found in diabetic glomerular disease. Proc. Natl. Acad. Sci. USA. 91:9436-9440.

9. Stitt, A.W., et al. 1997. Advanced glycation end products (AGEs) co-localize with AGE receptors in the retinal vasculature of diabetic and of AGEinfused rats. Am J. Pathol. 150:523-531.

10. Schmidt, A.M., et al. 1992. Isolation and characterization of two binding proteins for advanced glycosylation end products from bovine lung which are present on the endothelial cell surface. J. Biol. Chem. 267:14987-14997.

11. Neeper, M., et al. 1992. Cloning and expression of a cell surface receptor for advanced glycosylation end products of proteins. J. Biol. Chem. 267:14998-15004.

12. Yamagishi, S., et al. 1997. Advanced glycation end products-driven angiogenesis in vitro. Induction of the growth and tube formation of human microvascular endothelial cells through autocrine vascular endothelial growth factor. J. Biol. Chem. 272:8723-8730.

13. Yamagishi, S., Fujimori, H., Yonekura, H., Yamamoto, Y., and Yamamoto, H. 1998. Advanced glycation endproducts inhibit prostacyclin production and induce plasminogen activator inhibitor-1 in human microvascular endothelial cells. Diabetologia. 41:1435-1441. 
14. Yamagishi, S., et al. 1995. Receptor-mediated toxicity to pericytes of advanced glycosylation end products: a possible mechanism of pericyte loss in diabetic microangiopathy. Biochem. Biophys. Res. Commun. 213:681-687.

15. Tsuji, H., et al. 1998. Ribozyme targeting of receptor for advanced glycation end products in mouse mesangial cells. Biochem. Biophys. Res. Commun. 245:583-588.

16. Takamura, T., et al. 1998. Transgenic mice overexpressing type 2 nitricoxide synthase in pancreatic $\beta$ cells develop insulin-dependent diabetes without insulitis. J. Biol. Chem. 273:2493-2496.

17. Nakamura, S., et al. 1997. Progression of nephropathy in spontaneous diabetic rats is prevented by OPB-9195, a novel inhibitor of advanced glycation. Diabetes. 46:895-899.

18. Ronicke, V., Risau, W., and Breier, G. 1996. Characterization of the endothelium-specific murine growth factor receptor-2 (Flk-1) promoter. Circ. Res. 79:277-285.

19. Uchida, K. 1992. Recombination and amplification of multiple portions of genomic DNA by a modified polymerase chain reaction. Anal. Biochem. 202:159-161.

20. Sugaya, K., et al. 1994. Three genes in the human MHC class III region near the junction with the class II: gene for receptor of advanced glycosylation end products, PBX2 homeobox gene and Notch homolog, human counterpart of mouse mammary tumor gene int-3. Genomics. 23:408-419.

21. Tabata, H., Kubo, M., Suzuki, H., and Matsuzawa, T. 1998. Rapid determination of haemoglobin A1c and glucose in mice: strain differences, glucose tolerance tests and neonatal streptozotocin-induced diabetic model. Comparative Haematology International. 8:53-57.

22. Yamamoto, Y., Yamagishi, S., Hsu, C.C., and Yamamoto, H. 1996 Advanced glycation endproducts-receptor interactions stimulate the growth of human pancreatic cancer cells through the induction of platelet-derived growth factor-B. Biochem. Biophys. Res. Commun. 222:700-705.

23. Assmann, K.J.M., van Son, J.P.H.F., and Koene, R.A.P. 1991. Improved method for the isolation of mouse glomeruli. J. Am. Soc. Nephrol. 2:944-946.

24. Takasawa, S., et al. 1998. Cyclic ADP-ribose and inositol 1,4,5-trisphosphate as alternate second messengers for intracellular $\mathrm{Ca}^{2+}$ mobilization in normal and diabetic $\beta$-cells. J. Biol. Chem. 273:2497-2500.

25. Dong, Q.G., et al. 1997. A general strategy for isolation of endothelial cells from murine tissues. Characterization of two endothelial cell lines from the murine lung and subcutaneous sponge implants. Arterioscler. Thromb. Vasc. Biol. 17:1599-1604.

26. Brock, T.G., et al. 1996. Effects of granulocyte-macrophage colony-stimulating factor on eicosanoid production by mononuclear phagocytes. $J$. Immunol. 156:2522-2527.

27. Bradford, M.M. 1976. A rapid and sensitive method for the quantitation of microgram quantities of protein utilizing the principle of protein-dye binding. Anal. Biochem. 72:248-254.

28. Takeuchi, M., Makita, Z., Yanagisawa, K., Kameda, Y., and Koike, T. 1999. Detection of noncarboxymethyllysine and carboxymethyllysine advanced glycation end products (AGE) in serum of diabetic patients. Mol. Med. 5:393-405.

29. Bonsnes, R.W., and Taussky, H.H. 1945. On the colorimetric determination of creatinine by the Jaffe reaction. J. Biol. Chem. 158:581-591.

30. Doi, T., et al. 1990. Glomerular lesions in mice transgenic for growth hormone and insulinlike growth factor-1. Relationship between increased glomerular size and mesangial sclerosis. Am. J. Pathol. 137:541-552.

31. Nahas, A.M.E., Bassett, A.H., Cope, G.H., and Carpentier, J.E.L. 1991. Role of growth hormone in the development of experimental renal scarring. Kidney Int. 40:29-34.

32. Doi, T., et al. 1988. Progressive glomerulosclerosis develops in transgenic mice chronically expressing growth hormone and growth hormone releasing factor but not in those expressing insulin like growth factor-1. Am. J. Pathol. 131:398-403.

33. Kim, S., et al. 1995. Angiotensin II type 1 receptor blockade inhibits the expression of immediate-early genes and fibronectin in rat injured artery. Circulation. 92:88-95.

34. Doi, T., et al. 1991. Glomerulosclerosis in mice transgenic for growth hormone. Increased mesangial extracellular matrix is correlated with kidney mRNA levels. J. Exp. Med. 173:1287-1290.

35. Yamamoto, H., Uchigata, Y., and Okamoto, H. 1981. Streptozotocin and alloxan induce DNA strand breaks and poly(ADP-ribose) synthetase in pancreatic islets. Nature. 294:284-286.

36. Okamoto, H. 1990. The molecular basis of experimental diabetes. In Molecular biology of the islets of Langerhans. H. Okamoto, editor. Cambridge University Press. Cambridge, United Kingdom. 209-231.

37. Mogensen, C.E. 1999. Microalbuminuria, blood pressure and diabetic renal disease: origin and development of ideas. Diabetologia. 42:263-285

38. Tanaka, N., et al. 2000. The receptor for advanced glycation endproduct is induced by the glycation products themselves and TNF- $\alpha$ through $\mathrm{NF}-\kappa \mathrm{B}$, and by $17 \beta$-estradiol through $\mathrm{Sp}-1$ in human vascular endothelial cells. J. Biol. Chem. 275:25781-25790.

39. Brett, J., et al. 1993. Survey of the distribution of a newly characterized receptor for advanced glycation end products in tissues. Am. J. Pathol. 143:1699-1712.

40. Abel, M., et al. 1995. Expression of receptors for advanced glycosylated end-products in renal disease. Nephrol. Dial. Transplant. 10:1662-1667.

41. Tanji, N., et al. 2000. Expression of advanced glycation end products and their cellular receptor RAGE in diabetic nephropathy and nondiabetic renal disease. J. Am. Soc. Nephrol. 11:1656-1666.

42. Miyata, T., et al. 1996. The receptor for advanced glycation end products (RAGE) is a central mediator of the interaction of AGE-beta 2 microglobulin with human mononuclear phagocytes via an oxidant-sensitive pathway. Implications for the pathogenesis of dialysis-related amyloidosis. $J$. Clin. Invest. 98:1088-1094.

43. Connolly, D.T. 1991. Vascular permeability factor: a unique regulator of blood vessel function. J. Cell. Biochem. 47:219-223.

44. Schmidt, A.M., et al. 1994. Receptor for advanced glycation end products (AGEs) has a central role in vessel wall interactions and gene activation in response to circulating AGE proteins. Proc. Natl. Acad. Sci. USA. 91:8807-8811.

45. Horie, K., et al. 1997. Immunohistochemical colocalization of glycoxidation products and lipid peroxidation products in diabetic glomerular lesions. Implication for glycoxidative stress in the pathogenesis of diabetic nephropathy. J. Clin. Invest. 100:2995-3004.

46. Doi, T., et al. 1992. Receptor-specific increase in extracellular matrix production in mouse mesangial cells by advanced glycosylation end products is mediated via platelet-derived growth factor. Proc. Natl. Acad. Sci. USA. 89:2873-2877.

47. Iehara, N., Takeoka, H., Yamada, Y., Kita, T. and Doi, T. 1996. Advanced glycation end products modulate transcriptional regulation in mesangial cells. Kidney Int. 50:1166-1172.

48. Schmidt, A.M., et al. 1994. The endothelial cell binding site for advanced glycation end products consists of a complex: an integral membrane protein and a lactoferrin-like polypeptide. J. Biol. Chem. 269:9882-9888.

49. Hidaka, M., Stanford, W.L., and Bernstein, A. 1999. Conditional requirement for the Flk-1 receptor in the in vitro generation of early hematopoietic cells. Proc. Natl. Acad. Sci. USA. 96:7370-7375.

50. Hori, O., et al. 1995. The receptor for advanced glycation end products (RAGE) is a cellular binding site for amphoterin. Mediation of neurite outgrowth and co-expression of rage and amphoterin in the developing nervous system. J. Biol. Chem. 270:25752-25761.

51. Hofmann, M.A., et al. 1999. RAGE mediates a novel proinflammatory axis: a central cell surface receptor for S100/calgranulin polypeptides. Cell. 97:889-901.

52. Schafer, B.M., and Heinzmann, C.W. 1996. The S100 family of EF-hand calcium-binding proteins: functions and pathology. Trends Biochem. Sci. 21:134-140.

53. Park, L., et al. 1998. Suppression of accelerated diabetic atherosclerosis by the soluble receptor for advanced glycation endproducts. Nat. Med. 4:1025-1031. 